\title{
Cloud-shadow effects on the structure of the convective boundary layer
}

\author{
UlRich SCHUMANN*, ANDreas DöRnbraCK and BERnHARd MAYER
}

Institut für Physik der Atmosphäre, DLR, Oberpfaffenhofen, Germany

(Manuscript received June 22, 2001; in revised form October 29, 2001; accepted December 18, 2001)

\begin{abstract}
The influence of shadow from a broken cloud layer at top of a convective boundary layer (CBL) on the turbulent flow structure in the layer is investigated. The study addresses fair weather shallow convection over dry land surfaces as exemplified by a high-resolution satellite observation. A three-dimensional MonteCarlo radiative transfer calculation reveals the strong reduction of net solar flux in the shadow of such clouds with some enhancement between the clouds. The turbulent convection is studied using a simple conceptional model and a large eddy simulation. The models assume total shadow below clouds, instantaneous response of the vertical heat flux to shadow, and the kinematic association of clouds with updrafts in the upper CBL. The study shows that the convective motion is necessarily nonsteady in case of vertical shadow. With shadow the convective turbulent motions are of smaller scales compared to the reference case without shadow. Any asymmetry due to shadow for non-zero solar zenith angle has only very small impact on the turbulent motion field.

Zusammenfassung

Der Einfluss von Schatten einer durchbrochenen Bewölkung am Oberrand der konvektiven Grenzschicht auf die Struktur der Turbulenz in der Grenzschicht wird untersucht. Die Studie befasst sich mit der Konvektion bei Schönwetter über trockenen Landoberflächen wie beispielsweise in einem hochauflösenden Satellitenbild sichtbar. Eine drei-dimensionale Monte-Carlo Strahlungstransportrechnung zeigt die erwartete starke Reduktion des solaren Netto-Strahlungsflusses im Schatten und eine Zunahme der Strahlung zwischen den Wolken. Die turbulente Konvektion wird mit einem einfachen konzeptionellen Model und einer Grobstruktursimulation untersucht. Das Modell unterstellt totalen Schatten unterhalb der Wolken, sofortige Reaktion des vertikalen Wärmestroms auf Schatten und die unmittelbare Bildung von Wolken im oberen Teil der Aufwinde der konvektiven Grenzschicht. Es zeigt sich, dass die Konvektion bei vertikalem Schatten nicht stationär sein kann. Mit Schatten ist die konvektive Turbulenz kleinskaliger im Vergleich zum Referenzfall ohne Schatten. Unsymmetrien infolge Schattens bei schrägstehender Sonne haben nur einen sehr kleinen Einfluss auf das turbulente Bewegungsfeld.
\end{abstract}

\section{Introduction}

A layer of broken clouds at the top of the convective boundary layer (CBL) casts a spatially and temporally variable shadow. The shadow reduces the solar contribution to the heat budget at the surface and hence reduces the upward heat flux from the surface into the atmosphere. The change in heat flux causes a change in buoyancy inside the boundary layer which drives the turbulent convective motions and feedbacks on the formation of clouds and their shadows. This paper addresses the consequences of these interactions on the structure of turbulence in the CBL.

We expect the largest feedback of shadow on convection over homogeneous dry land surfaces, with low thermal inertia, for fair weather with weak mean wind. Over

\footnotetext{
* Corresponding author: Ulrich Schumann, Institut für Physik der Atmosphäre, Deutsches Zentrum für Luft- und Raumfahrt (DLR), Oberpfaffenhofen, Postfach 1116, 82230 Wessling, Germany, email: ulrich.schumann@dlr.de
}

oceans or wet land surfaces, the thermal response of the surface due to changes in solar flux by broken cloud layers would be small. For strong winds, the convective structure is mainly controlled by shear at the surface and wind reduces the impact of surface inhomogeneity on convection (HECHTEL et al., 1990). Therefore, this study concentrates on the case of a horizontally homogeneous adiabatic bottom surface at which the upward heat flux responds without any delay to changes in the solar radiation due to a broken cloud layer at the top of a shallow convective boundary layer. We do not study any longwave radiative effects. The study contributes to the understanding of the impact of temporally varying surface heat budgets on the structure of turbulence. It is known that convection reacts most strongly to surface inhomogeneities which have a horizontal scale of the same magnitude as the scale of convective motions itself (SCHUMANN, 1991). Since the scales of the shadow are clearly correlated with the scales of convection, a significant sensitivity of convection to shadow is to be ex- 
pected. One may even conceive the possibility that an inclined shadow for a sun outside the zenith causes a preference for convective circulation in a certain direction, which in turn could drive a horizontal motion either in the direction of or opposite to the shadow.

Surprisingly we found no study of this problem in the literature. Much is known about turbulent convection in the boundary layer, both for dry and cloudy conditions (see, e.g., DeardorfF, 1974; Stull, 1988; NieuWSTADT and DUYNKERKE, 1996; Plate et al. 1998; LOBOCKI, 2001). Several studies have considered stratocumulus convection over oceans which is driven radiatively by infrared cooling at cloud top (e.g., NICHOLLS and LeMONE, 1980; DEARdorfF, 1980; BRÜMMER et al., 1985; Nicholls, 1989; PENC and AlBRECHT, 1987; Plate et al., 1998; Moeng and SchumanN, 1991). Boundary layers with broken clouds have been observed over land (e.g. KIEMLE et al., 1997). However, the variation in upward heat flux from the surface due to shadow and its impact on the turbulent structure of convection has not yet been addressed. Certainly, it is a trivial observation that the short-wave flux below clouds is reduced compared with the non-cloudy case; this has effects on the local temperature, humidity, and even on aerosol properties (KAPUSTIN et al., 1974). Clouds influence the structure of convection as indicated by mesoscale clouds coinciding with the centre (closed cell) or the boundaries (open cell) of the convective cells at scales considerably larger than the boundary layer depth (ATKINSON and ZHANG, 1996; CHLOND and MÜLLER, 1998).

The turbulent structure in the dry CBL for weak mean winds over a homogeneous land surface is controlled by the depth of the boundary layer $(\mathrm{H})$ and the upward temperature or heat flux $Q=<w^{\prime} T^{\prime}>$ (here $w^{\prime}$ and $T^{\prime}$ represent the deviation of vertical velocity and temperature from the respective horizontal mean values; brackets $<>$ denote horizontal mean values). This flux induces a buoyancy forcing $\beta g Q$ of kinetic energy (with volumetric expansion coefficient $\beta=-1 / T_{0}$, absolute air temperature $T_{0}$, and gravity acceleration $\mathrm{g}$ ). The convective velocity field scales with $w^{*}=(\beta g Q H)^{1 / 3}$, the spatial structure of the convection scales with $z / H$ where $z$ is the height above the surface, and time and temperature scale with $t^{*}=H / w^{*}$ and $T^{*}=Q / w^{*}$ (DEARDORFF, 1970). The turbulence near the surface depends also on the surface roughness scale $z_{0}$ (MONIN and YAGLOM, 1971). At low mean wind speeds, the CBL develops a convective pattern with strong local updrafts surrounded by larger areas with slower sinking motions. This has important consequences for the vertical mixing processes (WILLIS and DEARDORFF, 1981; WYNGAARD and BROST, 1984; SCHMIDT and SCHUMANN, 1989, EBERT et al., 1989; SCHUMANN, 1989, 1993). In the horizontal, the size of convective cells scales with the depth of the boundary layer $\mathrm{H}$ but its actual size may become large depending on the boundary conditions (DÖRNBRACK, 1997).
In this paper, Section 2 discusses the problem by reference to a satellite observation and a radiation transfer computation of the short-wave flux reaching the surface under cloudy conditions. Section 3 describes a simple conceptual model which is used to show that shadow causes a non-steady response of convection to the shadow. Thereafter, we simulate shadow effects using a large eddy simulation (LES) method described in Section 4. The LES code MESOSCOP (SCHUMANN et al., 1987) has previously been applied to simulations of the CBL and compared with CBL simulations from other LES methods (NIEUWSTADT et al., 1993). The code has also been applied to other boundary-layer studies, including the convective boundary layer over an inclined surface (SCHUMANN, 1990), the neutral boundary layer (ANDRÉN et al., 1994), and a convective boundary layer over a wavy surface, both with zero and non-zero mean flow (KRETTENNAUER and SCHUMANN, 1992; DÖRNBRACK and SCHUMANN, 1993). Here, the LES is performed for a dry CBL in which the effects of shadow from clouds are included by assuming that clouds are present in the top part of updrafts within the CBL. This simplification may be justified as a first step towards the qualitative under-standing of the effects of shadow on turbulent convection. We show and discuss the LES results in Section 5, and in Section 6, we draw the conclusions.

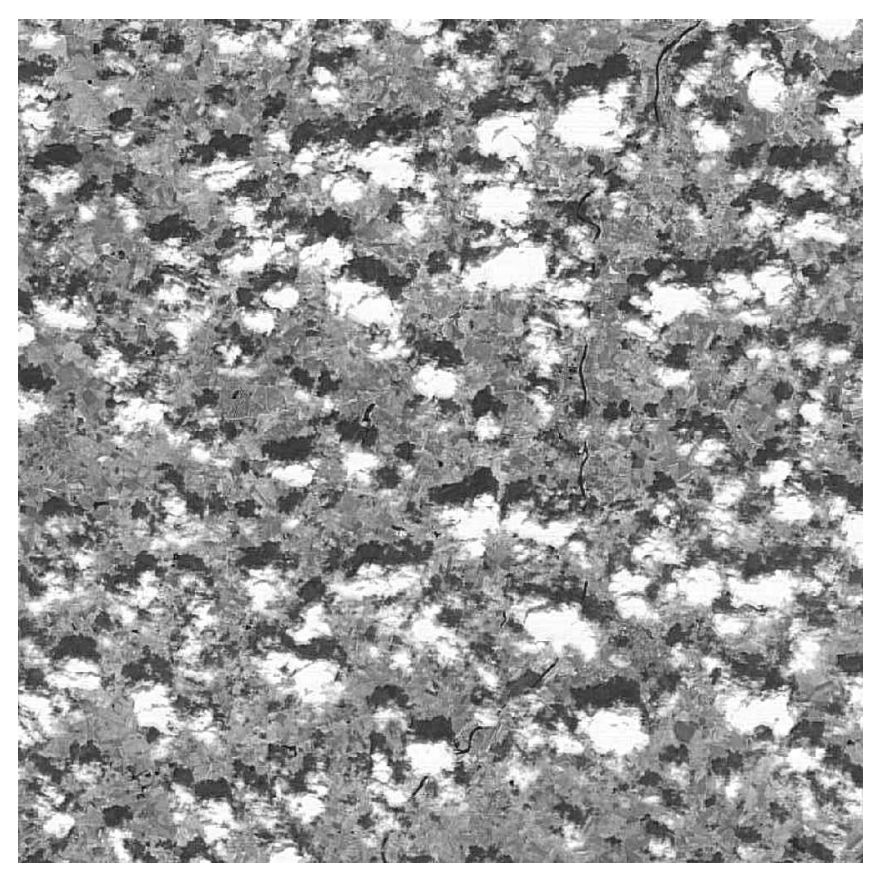

Figure 1: Broken clouds at top of a boundary layer over land as observed by the Modular Optoelectronic Multispectral Scanner (MOMS) onboard the Russian space station MIR over an area of $28 \times 28 \mathrm{~km}^{2}$ size, south-east of Poitiers, West France at 11 UTC 22 June 1998. North is toward the top of the figure (picture provided by A. DRESCHER, DLR). 

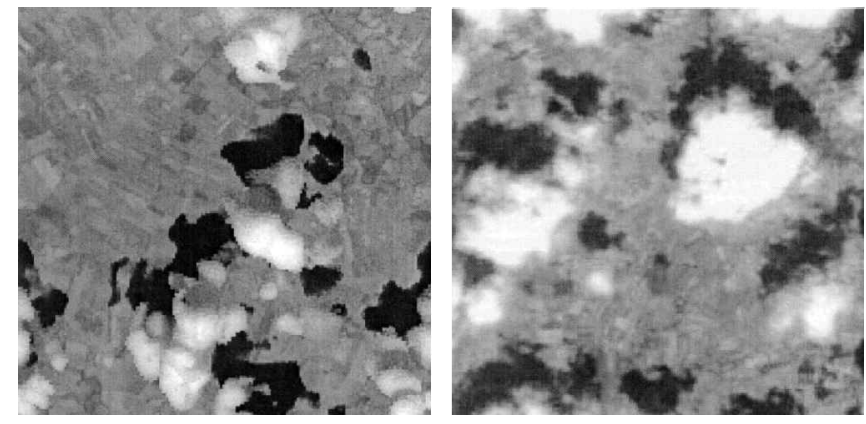

Figure 2: Nadir radiance as simulated by a three-dimensional radiative transfer code MYSTIC (left panel) compared to an observation by the MOMS satellite sensor (right panel). The size of both areas is $6.7 \times 6.7 \mathrm{~km}^{2}$. North is toward the top of the figures.

\section{Impact of convective clouds on short wave radiation reaching the surface}

The occurrence of broken clouds at top of the CBL is a common situation under fair weather conditions. For example, Fig. 1 shows a satellite picture of a layer of broken clouds at the top of a boundary layer over land, south-east of Poitiers, West France. The picture was taken shortly before noon in June. It covers a region of about $28 \times 28 \mathrm{~km}^{2}$, with a spatial resolution of $18 \mathrm{~m}$. White clouds are casting dark shadow regions to the north and below the clouds on the otherwise bright land surface. The size of the clouds is on the order of a few (1 to 3) kilometres horizontally, i.e. of the same magnitude or little larger than the expected depth $\mathrm{H}$ of a typical CBL over land at this time of the day.

Clouds generally increase the atmospheric albedo and reduce the amount of solar radiation reaching the surface. Hence, the surface radiation flux and consequently the mean amount of heat flux $Q$ driving the turbulent convection from the surface will be reduced in the presence of clouds. In addition, broken clouds cause small-scale variability in the surface flux. The surface radiation flux depends on the geometry of the clouds and the spatial distribution of the liquid water content and droplet size distribution within the clouds. These details are not known for the case shown in Fig. 1. For computation of the radiation field a three-dimensional radiative transfer model is required. The code MYSTIC (Monte Carlo code for the physically correct tracing of photons in cloudy atmospheres) can be applied for such purposes (MAYER, 1999; MAYER, 2000).

For illustration of the effects, Fig. 2 (top panel) shows a result of a simulation with MYSTIC of the radiation field in a cloud topped boundary layer with a shallow broken cumulus for which the liquid water content and droplet size distribution were taken from a previously performed large eddy simulation (STEVENS et al., 1998, 1999). The nadir radiance computed with MYSTIC for this case shows the bright cloud sides which are illuminated by the sun and the shadows to the north, qualitatively similar in structure to the satellite observation shown in Fig. 1 and repeated in comparable scales in Fig. 2 (right panel).

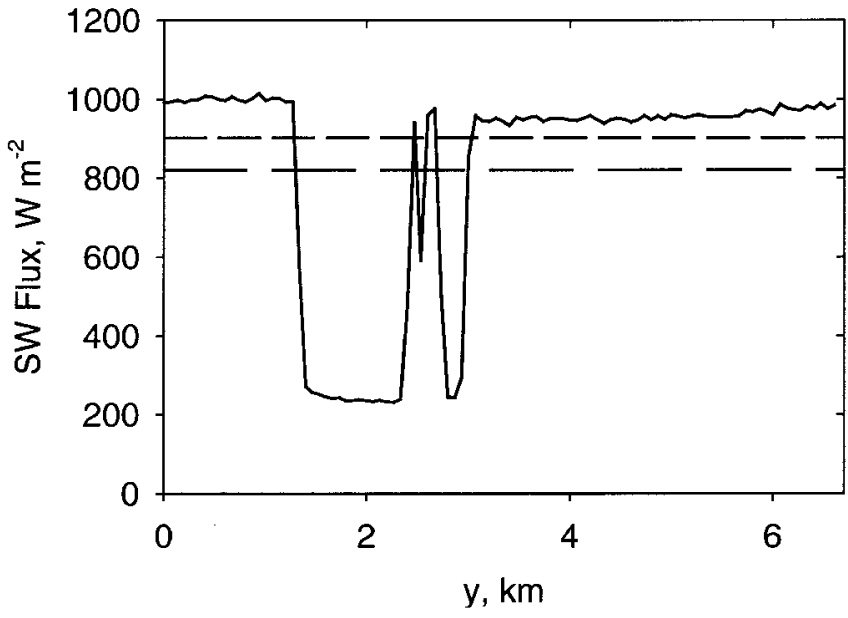

Figure 3: Integrated short-wave flux at the surface along a line at $x=2.5 \mathrm{~km}$ of the scene shown in the left panel of Fig. 2. The minima correspond to regions in the shadow of clouds. The horizontal lines denote the mean values with (long dashed) and without (shortdashed) clouds.

Fig. 3 shows the integrated short-wave surface flux along an arbitrary line through the LES results at the surface. In the shadow of the clouds, in particular below clouds with large water content, the amount of solar radiation reaching the surface is considerably reduced compared to non-shadow regions. The non-shadow regions in between the clouds receive more solar radiation due to scattering from illuminated cloud sides. In this particular example, the solar flux reaching the ground on average over the whole domain is reduced by about $10 \%$ due to increased albedo. In the shadow regions, the short-wave flux reaching the surface is reduced by about $75 \%$ compared to the cloudless value. Between the clouds the flux is increased to about 105 to $110 \%$ of the cloudless value. Increases to $125 \%$ and more have been observed (SEGAL and DAVIS, 1992). Both the shadows beside the clouds and the increase of the surface flux between the clouds are typical threedimensional phenomena.

The example shows that the radiation field can in principle be calculated with a three-dimensional radiative transfer model. However, such methods are very time-consuming. On a grid of $192 \times 192$ pixels, the calculation of integrated solar flux at the surface with a statistical uncertainty of $10 \%$ takes more than an hour of computational time on the current hardware (INTEL Pentium III, $600 \mathrm{MHz}$ ). With a small workstation cluster (16 or 32 processors), this number can be reduced to only a few minutes which is small enough to make a LES with a full 3D radiation calculation feasible. For the present study, however, a simplified model is used. The model assumes zero solar flux reaching the surface in the shadow of clouds and adjusts the flux between the cloud shadows such that the mean is a constant value $Q$. 


\section{Conceptual model}

In this paper, we do not simulate the details of clouds physics but simply assume that clouds form in the upper part of the CBL in regions with upward motions and have the same density as clear air. This is a strong simplification because clouds form only after some time in an updraft and decay some time after the updraft has ceased, and cloud air differs in density from clear air. However, we assume that the simple concept suffices for a first study of shallow convection. Hence, we simulate kinematically the shadow from clouds in a CBL as sketched in Fig. 4. The figure shows a simple convective cell with a cloud forming in the top part of the updraft. This cloud causes a shadow, which is shifted horizontally relative to the cloud's position. The horizontal shift depends on the boundary layer height and the solar zenith angle.

We distinguish three cases: A - no clouds and no shadows; B - clouds and inclined shadow; and C clouds with vertical shadow. Case A without clouds is the reference case for which the heat flux from the surface is $Q_{0}$. In cloudy cases, the mean surface heat flux $Q$ is usually less than $Q_{0}$, and this may cause different boundary layer depths. In this study the results are presented normalised with the actual surface heat flux $Q$ and boundary layer depth $H$ so that these parameters do not enter the simulations.

Case B (with clouds and inclined shadow) allows for situations where the surface directly below the cloud receives a flux $Q+q$ larger than the mean while the downdraft area receives a flux $Q-q$, less than average heat flux. Here $q$ is the disturbance relative to the horizontal mean. The case B depicted in Fig. 4 is special in that the inclined shadow coincides with the down-draft area and as such may enhance the convective cell. Case $\mathrm{C}$ with clouds and a vertical shadow is another special case. In this situation, the cloud shadow cuts off its own driving force by reducing the buoyancy gained from the surface heat flux. Other geometric configurations will occur

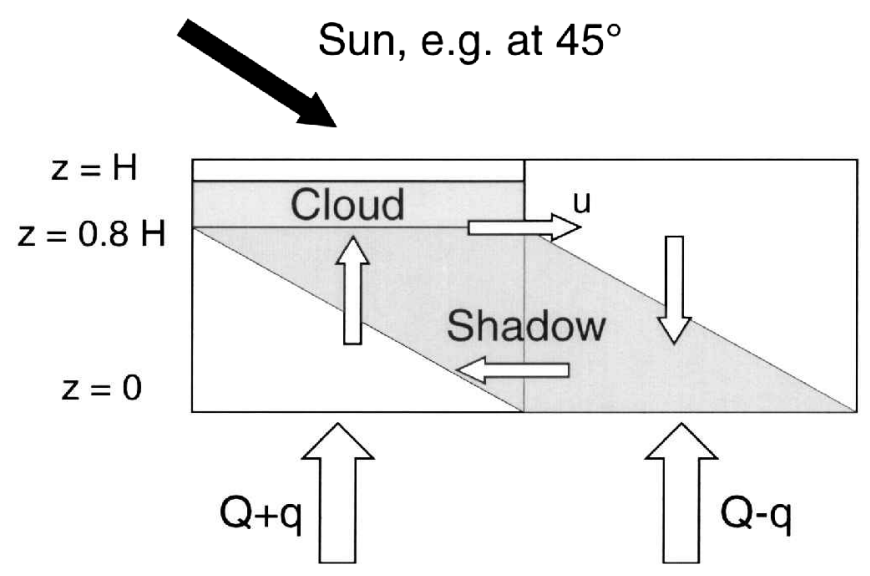

Figure 4: Model concept of convection with cloud formation in the top part of the updraft and shadows for $45^{\circ}$ inclination. in reality with intermediate inclinations and other vertical and horizontal scales, but the presented cases are sufficient to demonstrate that shadow may in principle enhance or reduce convection. For quadratic cells, solar zenith angles in the range from 0 to $45^{\circ}$ might be modelled by varying the value of $\mathrm{q}$ in the range from $-Q$ to $+Q$.

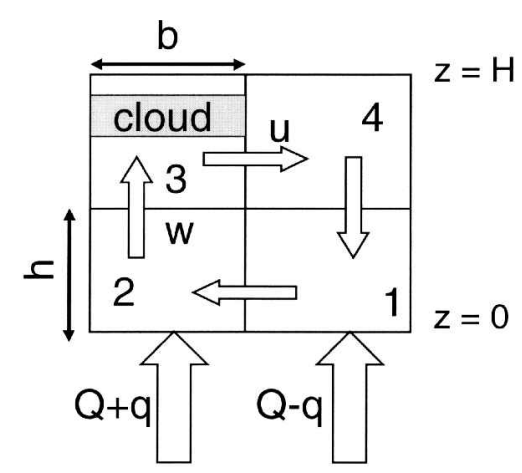

Figure 5: Four grid cell configuration in a vertical plane with mean circulation and surface heating, assuming a cloud in the top part of the updraft in cell 3.

To simulate these situations with a most simple conceptual model, we use the configuration with four grid cells sketched in Fig. 5. The equations of motion for this case are the same as derived in detail in SCHUMANN (1991). We do not repeat that derivation but explain the structure of the equations: The temperature $T_{1}$ in grid cell 1 satisfies a heat budget with horizontal and vertical advection of heat with resolved and subgrid scale speeds $u$ and $u^{\prime}$ and $w$ and $w^{\prime}$ and heat source $Q+q$,

$$
\begin{aligned}
V d T_{1} / d t= & -\left(u+u^{\prime}\right) h\left(T_{2}-T_{1}\right) \\
& -\left(w+w^{\prime}\right) b\left(T_{1}-T_{4}\right)+(Q+q) b
\end{aligned}
$$

and similarly for the other grid cells with temperatures $T_{2}, T_{3}$, and $T_{4}$. Here $h=H / 2$ is the height of the grid cells, $b$ is the width of the cells, $V=h b$ is the cell volume (per unit length in the third dimension), $u$ is the horizontal velocity, $w$ is the vertical velocity $(u h=w b$ for continuity), $u^{\prime}$ and $w^{\prime}$ are velocity scales representing turbulent velocity fluctuations causing the subgrid scale mixing in horizontal and vertical directions, respectively, across the surfaces between the grid cells, $Q$ is the mean heat flux and $q$ the heat flux disturbance due to shadow at the surface of grid cell number 1 . The subgrid velocity scales $u^{\prime}$ and $w^{\prime}$ are different in the two directions and are determined from a common isotropic turbulent velocity scale $v^{\prime}=\left(3 E_{s g s} / 2\right)^{1 / 2}$, as a function of the kinetic energy $E_{s g s}$ of the subgrid scale turbulent motions. The latter is determined by integrating a separate budget equation which includes the production rate of turbulence energy by shear, the buoyancy forcing and the dissipation of kinetic energy by small-scale motion inside the grid cells. Details are given in Eqns. 18, and 49 to 51 of SCHUMANN (1991). 


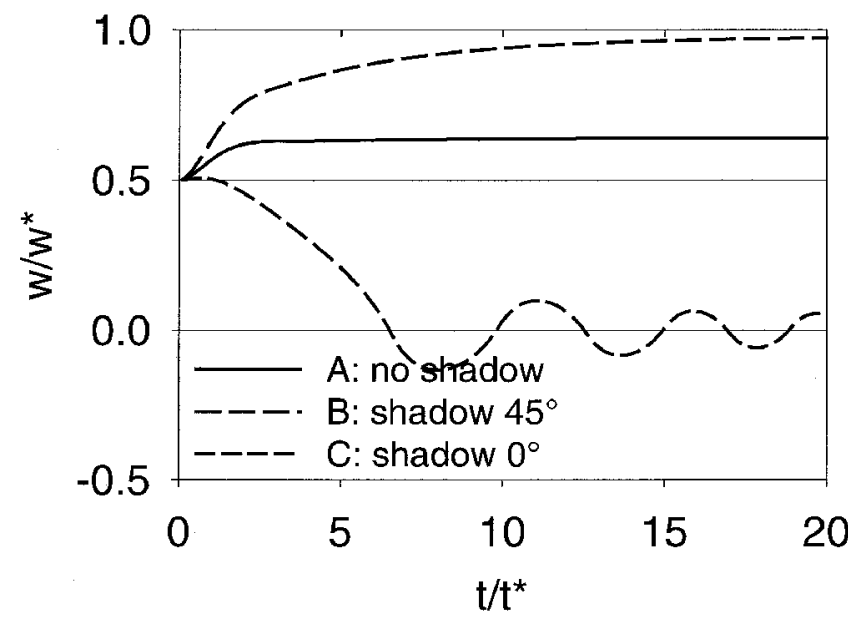

Figure 6: Normalised updraft velocity $w / w^{*}$ in the conceptual model versus time $t / t^{*}$ in case A: no shadow, B: shadow with $45^{\circ}$ inclination, $\mathrm{C}$ : vertical shadow. The scales $w^{*}$ and $t^{*}=H / w^{*}$ are formed with the mean surface heat flux $Q$ applying for each case.

After elimination of vertical velocity and pressure from the equations of motion using the continuity equation (SCHUMANN, 1991), the remaining equation of motion for horizontal velocity $u$ between the cells is given by

$$
\begin{aligned}
V & \left(1+\frac{h^{2}}{b^{2}}\right) d u / d t \\
= & \beta g\left(T_{2}+T_{3}-T_{1}-T_{4}\right) \frac{h^{2}}{2} \\
& \quad-\left(4 u^{\prime} h^{3} / b^{3}+4 w^{\prime} b\right) u-u^{* 2} b .
\end{aligned}
$$

The first term includes the effective mass of the fluid due to horizontal and vertical acceleration, the second term is the buoyancy forcing, the third term describes subgrid scale mixing of momentum and the last term friction at the surface. Here $u^{*}$ is the surface friction velocity which is computed from $u, Q$, and surface roughness $z_{0}$ according to the Monin-Obukhov relationships (SCHUMANN, 1991).

The system has an analytical steady-state solution (SCHUMANN, 1991), but knowledge of the steady solutions is not sufficient for this study. For the non-steady case, the 6 coupled ordinary differential equations for $T_{1}, T_{2}, T_{3}, T_{4}, u$ and $E_{s g s}$ are integrated numerically. The non-steady model is applied for cases $\mathrm{A}, \mathrm{B}$ and $\mathrm{C}$, with $q=0$ for $\mathrm{A} ; q=Q$ for $\mathrm{B}$ and $q=-Q$ for $\mathrm{C}$. In all three cases we set $z_{0} / H=10^{-4}$, and set the horizontal scale to $b=2 H$, because a horizontally inhomogeneous surface heat flux causes the largest effect on the convection for $b$ close to this value (SCHUMANN, 1991).

Fig. 6 shows the results for vertical velocity for the three cases A, B, and C. The plot shows the normalised velocities. In all three cases the velocity scale is $w^{*}=(\beta g Q H)^{1 / 3}$. The normalised asymptotic values for cases $\mathrm{A}$ and $\mathrm{B}$ are $w / w^{*}=0.64$ and 0.98 , respectively. Note that the value of $w^{*}$ is computed with the average heat flux $Q$ reaching the surface. If the surface heat flux $Q$ in the cloudy case would be $50 \%$ of the noncloud value $Q_{0}$, the velocity scale $w^{*}$ would be a factor of 0.79 times smaller than $w_{0}^{*}=\left(\beta g Q_{0} H\right)^{1 / 3}$, because of the third power dependence of $w^{*}$ on $Q$. For this case, the vertical velocity is $0.78 w_{0}^{*}$ for case $\mathrm{B}$ and $0.64 w_{0}^{*}$ for case A. Hence the vertical velocity in case B is larger than for case A also when accounting for a factor of 2 reduction of $Q$ by the cloud shadow. The convection with shadow is stronger than without shadow because the solar heating effectively coincides with the convective structure.

Case C (vertical shadow) produces motions with smaller amplitudes and shows an oscillating but decaying vertical velocity. This decaying oscillation is caused by a sequence of effects: As soon as an updraft and a cloud form, the shadow shuts off the initial surface heating driving the convection. As a consequence, the air in the updraft cools and after some time the air in the updraft gets cooler than in the downdraft. The resultant buoyancy force decelerates the convective circulation. After some time, the circulation changes sign because of negative buoyancy in the former updraft. Then an updraft and a cloud form in the neighbouring cell causing the same sequence of effects with opposite sign but at smaller amplitude and at shorter time scales. For the present case with zero heat flux from the surface in the shadow, a state is eventually approached with high frequency velocity sign changes and near zero velocity magnitude. In other words, for complete vertical shadow, no meaningful steady state with non-zero velocity exists. Although these are highly simplified situations, this simple analysis shows that the turbulent convection for shadowed cases has smaller time scales than the convection in the non-shadow case.

\section{Large eddy simulations model}

We consider a domain of horizontal size $L_{x}=L_{y}=12 \mathrm{H}$, and a vertical size of $L_{z}=H$. The details of the top boundary are not considered to be important. We assume for simplicity an adiabatic free-slip top boundary which approximates a strong inversion layer at top of the domain. This is done because in this case we do not need to simulate the flow fields in the atmosphere above the CBL. The bottom surface is taken as a spatially homogeneous rough surface with roughness $z_{0}$. In the reference cases, $z_{0} / H=10^{-4}$, which is also the reference value used in previous simulations (SCHMIDT and SCHUMANN, 1989). Clouds are assumed to form at $z=0.8 H$ when $w>0$. This is the easiest way to represent cloud shadows in the model, but is admittedly a farreaching simplification. For the case with a zero shadow inclination angle, the pattern of the shadow at the surface is just the same as the pattern of the field with $w>0$ at $z / H=0.8$. For a $45^{\circ}$ inclination, the shadow pattern is shifted by $0.8 H$ into positive x-direction. At the bottom surface, the vertical heat flux is positive and spatially constant in grid cells belonging to sunny regions and 

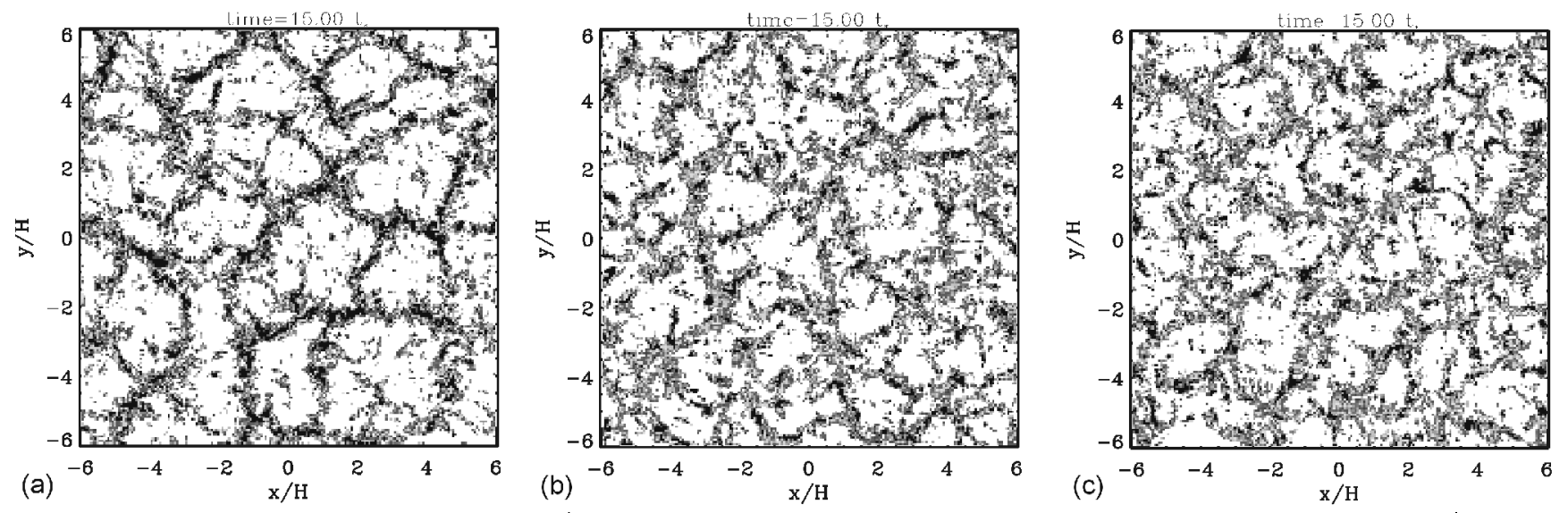

Figure 7: Lines of constant vertical velocity $w / w^{*}$ versus horizontal coordinates $x / H$ and $y / H$ at altitude $z / H=0.25$, and time $t / t^{*}=15$ of the LES. a) case A, without clouds; b) case $\mathrm{B}$, shadow at $45^{\circ}$; c) case $\mathrm{C}$, shadow at $0^{\circ}$.

zero in grid cells belonging to the shadow region vertically (case C) or inclined (case B) below the "clouds". The heat flux in the sunny regions is determined such that the average over the whole surface equals the constant value $Q$. The flux in the sunny region varies slightly with time depending on the area fraction covered by updrafts but approaches a constant value in the final quasisteady state. The zero heat flux in the shadow regions implies total darkness. This assumption is made to reduce the number of parameters in this explorative study. For the same reason, we assume zero mean wind. Laterally, the LES code assumes periodic boundary conditions in both horizontal directions with equal periodicity lengths $L_{x}$ and $L_{y}$.

The LES is performed using grid spacings $\Delta_{x}=\Delta_{y}=$ $\Delta_{z}=H / 16$, i.e. with $192 \times 192 \times 16$ grid cells. The time step is set to about $0.01 t^{*}$ as required for numerical stability of the explicit integration scheme. Initial conditions describe constant mean fields with small random disturbances. The computed flow fields are nonsteady from the beginning and do not become steady in its local values. The simulations are integrated over a time period from 0 to $30 t^{*}$, which is a long period compared to the turn-over time scale of the largest convection cells (about $3 t^{*}$ ). This is done to make sure that any statistics computed at later times from the three-dimensional nonsteady motion fields represent the quasi-steady statistics of the turbulent motions.

\section{Large-eddy simulation results}

We present simulation results for the three cases as before: A - no shadow, B - shadow with $45^{\circ}$ zenith angle, C - vertical shadow. Fig. 7 shows the vertical velocity $w / w^{*}$ in a horizontal plane versus $x$ and $y$ at $z / H=0.25$ at $t / t^{*}=15$ of the LES for the three cases. We see turbulent structures with different horizontal scales which will be quantified using spatial correlation analysis later. It appears that with shadow, the vertical motion fields exhibit shorter horizontal scales than without shadow. The results for cases $\mathrm{B}$ and $\mathrm{C}$ are similar without any major difference in the structure and scales of turbulence.

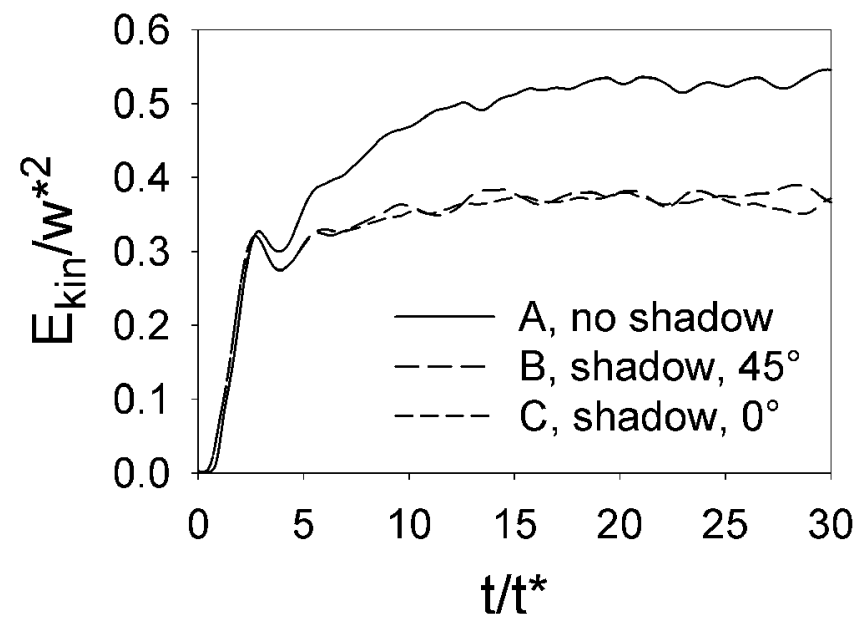

Figure 8: Mean total kinetic energy $\mathrm{E}_{\mathrm{kin}}=\left(\left\langle u^{\prime} u^{\prime}\right\rangle+\left\langle v^{\prime} v^{\prime}\right\rangle\right.$ $\left.+\left\langle w^{\prime} w^{\prime}\right\rangle\right) / 2$ in the computational domain of the LES versus time for cases $\mathrm{A}$ to $\mathrm{C}$.

Fig. 8 shows the average mean total kinetic energy $E_{k i n}$, which is the sum of kinetic energy of motions at resolved and at subgrid scales, averaged over the whole CBL from bottom to top and over the whole domain laterally, versus time. After a transition from near zero the averaged kinetic energy approaches a quasi steady state at times larger than about $15 t^{*}$. The energy is largest for the case without shadow (A), and smaller for the two cases (B and C) with shadow of different inclination. Hence, in contrast to the conceptional model, the LES results do not show stronger convective motions in the case with inclined shadow compared with the case without clouds. This different result might be caused by a less coherent motion structure in the LES compared to that assumed in the conceptional model. For case B, configurations in which the sun just shines below an updraft and enhances the upward motion occur only occasionally within the LES but were assumed to be present always in the simple model.

The different mean kinetic energies in cases A to $\mathrm{C}$ depicted in Fig. 8 imply different length scales: The kinetic energy $E_{k i n}$ of the turbulent motions in the boundary layer is created by buoyancy flux $\beta g\left\langle w^{\prime} T^{\prime}\right\rangle$ and 

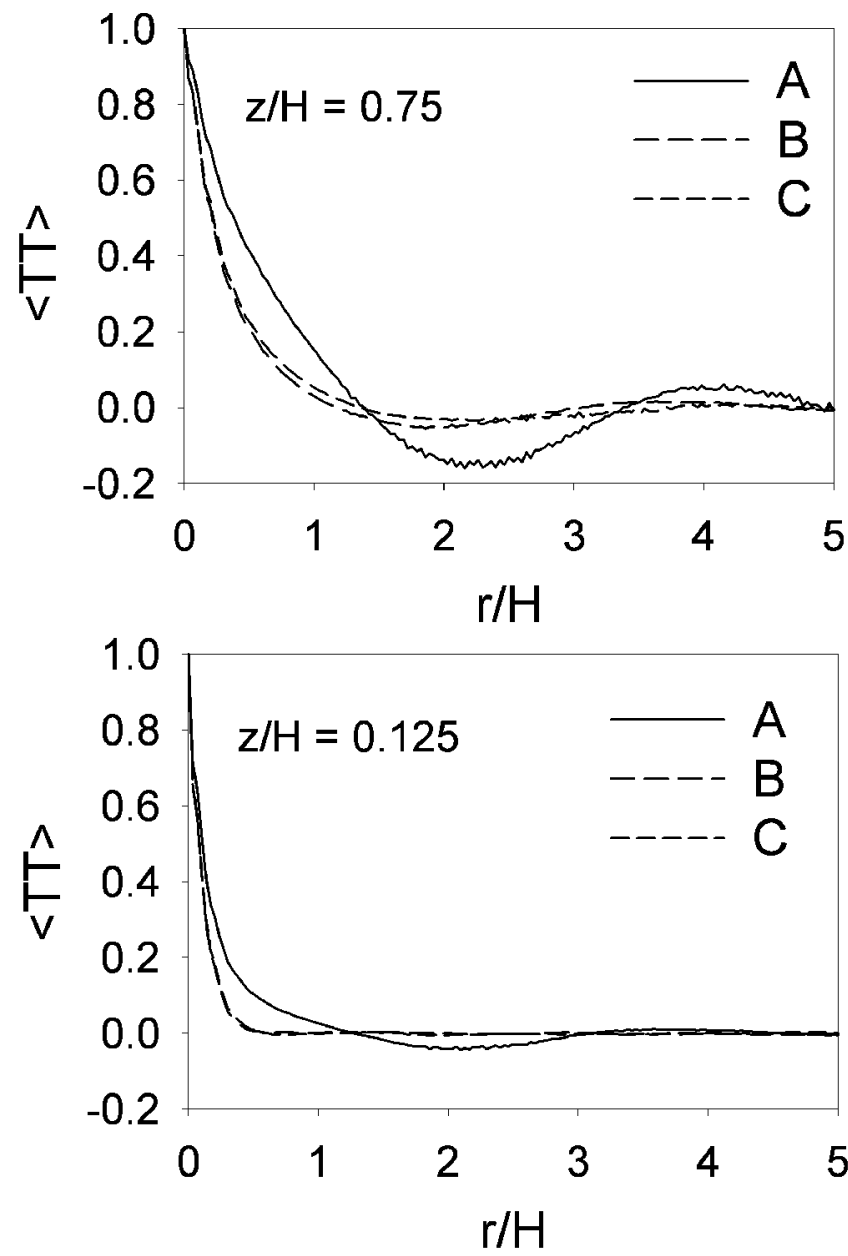

Figure 9: Normalised horizontal correlation between temperature fluctuations at any two positions in a horizontal plane separated horizontally by a distance $r / H$ at two altitude levels $z / H=0.125$ and 0.75 in the LES for the three cases A, B, and C.

dissipated by turbulent dissipation $\varepsilon$. Shear plays no role for the mean kinetic energy in this case because of zero mean wind with zero mean shear. Hence, the total kinetic energy, integrated over the whole domain, satisfies

$$
\int\left(d E_{k i n} / d t\right) d z=\int \beta g<w^{\prime} T^{\prime}>d z-\int \varepsilon d z .
$$

In the horizontal mean, the buoyancy flux $\beta g$ $\left\langle w^{\prime} T^{\prime}\right\rangle$ is the same for all cases - linearly decreasing from the positive surface value at the bottom to zero at the top boundary. Hence, in steady state, the integral of $\varepsilon$ must also be the same in all simulations. According to the classical inertial subrange concept (KOLMOGOROV, $1941), \varepsilon$ scales with kinetic energy $E_{k i n}$ and a suitable integral length scale of turbulence $L$ as

$$
\varepsilon=E_{k i n}{ }^{3 / 2} / L \text {. }
$$

Hence, different levels of $E_{k i n}$ imply different sizes of the length scale $L$ of turbulence. The results shown in Fig. 8 imply smaller length scales $L$ for the cases with shadow than in the reference case. The cases B and $\mathrm{C}$ show no difference as a function of shadow inclination. This conclusion is consistent with the structure observed in Fig. 7. The tendency of shadow to cause smaller length scales of motion structures can also be supported by analysis of horizontal correlation scales such as derived from the correlation coefficient,

$$
<T T>(z, r)=<T^{\prime}(x, y, z, t) T^{\prime}(x+r, y, z, t)>\text {. }
$$

Fig. 9 shows the correlation coefficient for $z=$ $0.125 \mathrm{H}$ and $z=0.75 \mathrm{H}$ versus the horizontal distance $\mathrm{r}$ between the two points in one horizontal plane for which the temperature correlation is computed. The correlation is largest (equal to the local variance) for $r=0$ and decays with growing distance $r$, gets negative when correlating warm updraft air with cold downdraft air and vice versa and decays to zero at large distance $r$. The distance $r$ where the correlation decays to a certain fraction (say 50\%) of its value at $r=0$ can be interpreted as a length scale of turbulence. We see that the correlation decreases to about $50 \%$ of its initial value at a distance $r$ which is about $20-40 \%$ shorter in cases with shadow than in the reference case without shadow, and decreases over shorter scales with $r$ at the lower altitudes than at higher altitudes in the CBL. Hence, the turbulence with shadow is formed by temperature variations of smaller scales than without shadow, in particular at lower altitude.

Fig. 10 shows the results of the LES in terms of horizontally averaged turbulence variances and temperature fluxes for the cases under consideration. The results for the reference case A are as described in KRETTENAUER and SCHUMANN (1992) for the same boundary conditions. The temperature flux decays linearly in all cases from the surface value $Q$ to zero at the adiabatic top. The small deviation from the linear shape in the first grid cell is unimportant; it is caused by using different numerical approximations in analysing the fluxes compared to those used in the numerical integration scheme (SCHMIDT and SchumAnN, 1989). The horizontal velocity fluctuations are largest near the bottom and top surfaces because these barriers convert vertical motions into horizontal ones. The horizontal velocity fluctuations are smaller by about $20-40 \%$ in cases with shadow than in the case without shadow. This is in line with enhanced dissipation by small-scale motions inside the flow and by surface friction at the lower boundary. The vertical velocity variance is largest in the middle of the CBL, as expected because of strongest updrafts at those levels, and slightly larger in the bottom part of the CBL than in the top part of the CBL because of larger buoyancy flux, causing a stronger buoyancy forcing of kinetic energy in the lower part. With shadow, the vertical velocity variance is smaller mainly because of smaller convective scales causing larger internal dissipation. Surface friction does not play an important role for the vertical velocity variance. The reduction in vertical variance is larger in the upper part than in the lower part of the CBL, which indicates that the shadows cause smaller scale vertical motions in particular in the upper CBL. The turbulence structure and statistics is found to be more or 

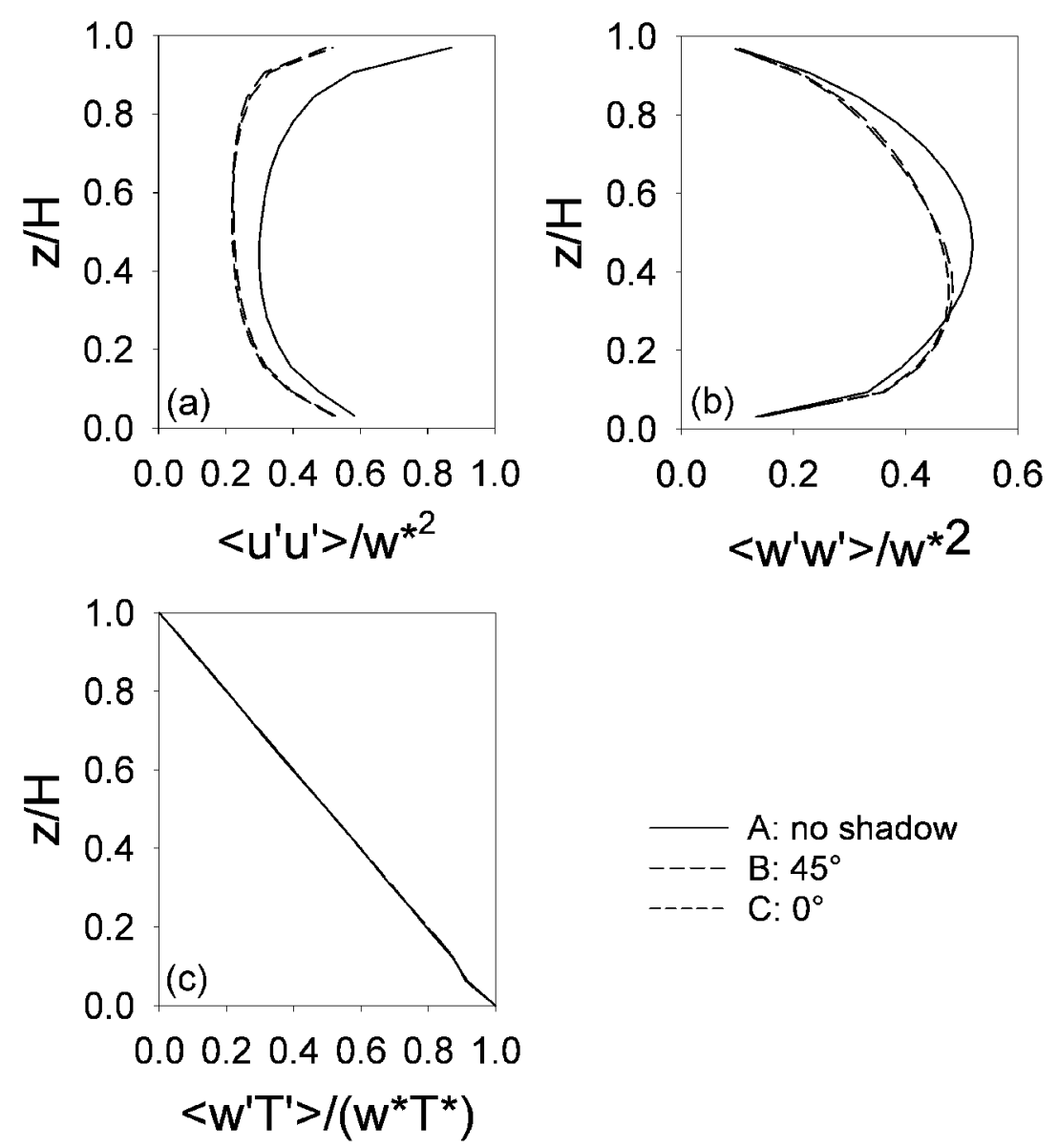
A: no shadow
B: $45^{\circ}$
C: $0^{\circ}$

Figure 10: Normalised vertical heat (temperature) flux $\left\langle w^{\prime} T^{\prime}\right\rangle$ and variances $\left\langle u^{\prime} u^{\prime}\right\rangle$ and $\left\langle w^{\prime} w^{\prime}\right\rangle$ of horizontal ( $x$-direction) and vertical velocity fluctuations versus altitude for the four cases simulated with the LES.

less the same for $45^{\circ}$ inclined and vertical shadow (cases $\mathrm{B}$ and $\mathrm{C}$ ). This indicates that the structure of the CBL is still mainly controlled by the internal dynamics of the CBL and not much by the coherent shift in shadow.

Finally we looked for the possibility that an inclined shadow induces a systematic motion into a horizontal direction. The solar heating does not cause a horizontal force directly. However, it appears conceivable that convection with inclined shadow prefers a certain circulation orientation relative to the sun. The mean circulation may drive a mean motion of the whole layer into one direction after momentum exchange with the surface. The momentum exchange depends on the surface roughness. However, the LES results do not show any significant mean motion, neither of the case B described above nor for a case with enhanced surface friction.

\section{Conclusions}

A first, explorative study of the influence of cloud shadow on the turbulent structure of the cloud-topped convective boundary layer has been presented. The model study is based on assumptions which need to be overcome in future studies if one is interested in more realistic cases. This is true in particular with the assumptions of total shadow and zero heat flux from the surface below clouds, instantaneous response of the vertical heat flux to shadow, the kinematic association of clouds with updrafts in the upper CBL, and the assumption that the buoyancy of the air depends only on the temperature and not on other cloud parameters such as liquid water content. Moreover, the CBL is treated as a uniform layer between a rough surface and an adiabatic free-slip upper boundary at fixed height. Hence, it does not consider the impact of cloud formation on the depth of the boundary layer. Finally the study assumes zero mean wind for which the impact of any inhomogeneity in surface fluxes on convection should be largest. However, the study allows one to draw some conclusions on the effects of temporally and spatially variable surface heat fluxes, caused by shadow, on the turbulent structure of the CBL.

The conceptual model shows that the convective motion is necessarily nonsteady in case of vertical shadow. Once updrafts and clouds form, they cause shadows which reduce their own driving buoyancy and hence, counteract their existence. If the shadow formed from a cloud in an updraft is inclined such that it reduces the surface heat flux just below the neighbouring downdraft, while the sun shines below the cloudy updraft, the shadow may enhance the circulation by heating below an updraft and cooling below a downdraft. However, this is the case only under very special conditions for a small 
subset of cloud structures. The LES study shows no major difference in the turbulence for the two inclination angles considered. Hence, such an enhancement of convection by shadow does not appear to be important.

The LES study shows convective structures that are clearly of smaller scales and appear to be more chaotic if shadow is included compared to the reference case with the same mean surface heat flux. This is reflected both by the integral length scale of dissipation and by horizontal correlation scales of motion and temperature fluctuations. We could not find a systematic mean drift induced by the inclined shadow.

The reduced scales found in convection with shadow and the enhanced scales which are known to occur with latent heat release in mesoscale convective cells (CHLOND and MüLLER, 1998) appear to be consistent: Whereas shadow causes a cooling of the air in the updraft below the clouds and hence a negative feedback on buoyancy forcing, latent heat release causes a warming of the updraft and hence a positive feedback on convection.

It would be of interest to study the correlation between vertical heat fluxes and shadow from observations. For example, how quickly does the vertical heat flux follow changes in shadow? Also it might be of interest to perform LES studies with more realistic cloud physics and a radiative transfer model (such as MYSTIC) in order to compute more realistic changes in the solar heat flux arriving at the surface as a function of cloudiness. The formation of liquid water in nonprecipitating clouds and the effect of latent heat release and the liquid water on buoyancy could be rather easily implemented assuming that all water vapour above saturation gets converted to liquid water (DEARDORFF, 1980; MoENG, 1986). For precipitating clouds the surface should become wet and shadow effects should be reduced because of smaller response of the heat flux from a wet surface compared to a dry one. Inclusion of such physics into the LES would make such studies far more computer time demanding. However, the results obtained so far show that the structure of the CBL may change considerably and the mean turbulence variances and scales may change by about $20-40 \%$, which may justify such refined studies in the future.

\section{Acknowledgements}

This paper is dedicated to Professor Dr. Hans HINZPETER (1921-1999). The members of the Institute of Atmospheric Physics of the DLR Oberpfaffenhofen are grateful for his major contributions to the development of research at the Institute over more than two decades, in particular initiating numerical and experimental studies of mesoscale and turbulent processes, shallow and deep convection, radiative transfer, and climate research. Moreover we thank Professor Dr. Hartmut GRASSL for inviting us to present this paper at the Colloquium in memoriam of Hans HINZPETER in
February 2001, two reviewers for very valuable comments, and Dr. Andreas CHLOND for editing this special issue.

\section{References}

Andrén, A., A.R. Brown, J. Graf, P.J. Mason, C.H. Moeng, F.T.M. NieuWSTAdT, U. SCHUMAnN, 1994: Large-eddy simulation of a neutrally stratified boundary layer: A comparison of four computer codes. - Quart. J. Roy. Meteor. Soc. 120, 1457-1484.

AtKinson, B.W., J.W. ZHANG, 1996: Mesoscale shallow convection in the atmosphere. - Rev. Geophys. 34, 403431.

BrüMmer, B., S. BAKAN, H. HinZPeter, 1985: KonTur: Observations of cloud streets and open structures. - Dyn. Atmos. Oceans 9, 281-296.

Chlond, A., G. Müller, 1998: Mesoscale cellular convection during cold air outbreaks: A numerical study. - In: Clear and Cloudy Boundary Layers, Holtslag, A.A.M., P.G. DUYNKER KE (Eds.), Royal Netherlands Academy of Arts and Sciences, 1000 GC Amsterdam, the Netherlands, ISBN 90-6984-235-1,219-239.

DEARDORFF, J.W., 1970: Convective velocity and temperature scales for the unstable boundary layer and for Rayleigh convection. - J. Atmos. Sci. 27, 1211-1213.

- 1974: Three-dimensional numerical study of the height and mean structure of a heated planetary boundary layer. - Bound.-Layer Meteorol. 7, 81-106.

- 1980: Stratocumulus-capped mixed layers derived from a three-dimensional model. - Bound.-Layer Meteorol. 18, 495-527.

DÖRNBRACK, A., 1997: Broadening of convective cells. Quart J. Roy. Meteor. Soc. 123, 829-848.

DÖRNBRACK, A., U. SCHUMANN, 1993: Numerical simulation of turbulent convective flow over wavy terrain. Bound.-Layer Meteorol. 65, 323-355.

Ebert, E.E., U. Schumann, R.B. STull, 1989: Nonlocal turbulent mixing in the convective boundary layer evaluated from large-eddy simulation. - J. Atmos. Sci. 46, 21782207.

Hechtel, L.M., C.-H. Moeng, R.B. Stull, 1990: The effects of nonhomogeneous surface fluxes on the convective boundary layer: A case study using large-eddy simulation. - J. Atmos. Sci. 47, 1721-1741.

Kapustin, V.N., Y.S. Lyubovtseva, G.V. RozenberG, 1974: Variability of aerosols under the influence of cloud modulation of the radiation field. - Izvestya, Atmospheric and Oceanic Physics 10, 819-821.

Kiemle, C., G. Ehret, A. Giez, K.J. Davis, D.H. LENSCHOW, S.P. ONCLEY, 1997: Estimation of boundary layer humidity fluxes and statistics from airborne differential absorption lidar (DIAL). - J. Geophys. Res. 102, 29,189-29,203.

Kolmogorov, A.N., 1941: The local structure of turbulence in incompressible viscous fluid for very high Reynolds number. - Dokl. Akad. Nauk. SSSR 30, 299-303.

Krettenauer, K., U. Schumann, 1992: Numerical simulation of turbulent convection over wavy terrain. - J. Fluid Mech. 237, 261-299.

LOBOCKI, L., 2001: Calculation of surface fluxes under convective conditions by turbulence closure models. - J. Appl. Meteor. 40, 604-621. 
MAYER, B., 1999: I3RC phase 1 results from the MYSTIC Monte Carlo model. - Intercomparison of 3D radiation codes, Tucson, AZ, Nov. 15-17, 1999. [Available from http://climate.gsfc.nasa.gov/[3RC/workshopreports.html]

- 2000: I3RC phase 2 results from the MYSTIC Monte Carlo model. - Intercomparison of $3 \mathrm{D}$ radiation codes, Tucson, AZ, Nov. 17-19, 2000.

MoENG, C.-H., 1986: Large-eddy simulation of the stratustopped boundary layer. Part I: structure and budgets. - J. Atm. Sci. 43, 2886-2900.

Moeng, C.-H., U. Schumann, 1991: Composite structure of plumes in stratus-topped boundary layers. - J. Atmos. Sci. 48, 2280-2291.

Monin, A.S., A.M. Yaglom, 1971: Statistical Fluid Mechanics: Mechanics of Turbulence, Vol. 1. - MIT Press, Cambridge, Mass, 769 pp.

NiCHOLLS, S., 1989: The structure of radiatively driven convection in stratocumulus. - Quart. J. Roy. Meteor. Soc. 115, 487-511.

Nicholls, S., M.A. LeMone, 1980: Fair weather boundary layer in GATE: the relationship of subcloud fluxes and structure to the distribution and enhancement of cumulus clouds. - J. Atmos. Sci. 37, 2051-2067.

Nieuwstadt, F.T.M., P.G. Duynkerke, 1996: Turbulence in the atmospheric boundary layer. - Atmos. Res. 40, $111-142$.

Nieuwstadt F.T.M., P.J. Mason, C.H. Moeng, U. SCHUMANN, 1993: Large-eddy simulation of the convective boundary layer: A comparison of four computer codes. - Turbulent Shear Flows 8, DURST, F., R. FRIEDRICH, B.E. Launder, F.W. Schmidt, U. Schumann, J.H. WhitelaW (Eds.), Springer, Berlin, 343-367.

Penc, R.S., B.A. Albrecht, 1987: Parametric representation of heat and moisture fluxes in cloud-topped mixed layers. - Bound.-Layer Meteorol. 38, 225-248.

Plate, E.J., E.E. Federovich, D.X. Viegas, J.C. WynGAARD (Eds.), 1998: Buoyant Convection in Geophysical Flows. - NATO ASI Series, Kluwer, Dordrecht, 491 pp.
SchmidT, H., U. SchumanN, 1989: Coherent structure of the convective boundary layer derived from large-eddy simulations. - J. Fluid Mech. 200, 511-562.

SCHUMANN, U., 1998: Minimum friction velocity and heat transfer in the rough surface layer of a convective boundary layer. - Bound.-Layer Meteorol. 44, 311-326.

- 1989: Large-eddy simulation of turbulent diffusion with chemical reactions in the convective boundary layer. - Atmos. Env. 23, 1713-1727.

- 1990: Large-eddy simulation of the up-slope boundary layer. - Quart. J. Roy. Meteorol. Soc. 116, 637-670.

- 1991: A simple model of the convective boundary layer over wavy terrain with variable heat flux. - Beitr. Phys. Atmosph. 64, 169-184.

- 1993: Transport asymmetry in skewed convective circulations. - J. Atmos. Sci. 50, 116-119.

Schumann, U., T. Hauf, H. Höller, H. Schmidt, H. VOLKERT, 1987: A mesoscale model for the simulation of turbulence, clouds and flow over mountains: formulation and validation examples. - Beitr. Phys. Atmosph. 60, 413446.

SEGAL, M., J. DAVIS, 1992: The impact of deep cumulus reflection on the ground-level global irradiance. - J. Appl. Met. 31, 217-222.

Stevens, B., W.R. Cotton, G. Feingold, C.-H. MoENG, 1998: Large-eddy simulation of strongly precipitating, shallow, stratocumulus-topped boundary layers - J. Atmos. Sci. 55, 3616-3638.

Stevens, B., C.-H. Moeng, P.P. Sullivan, 1999: Largeeddy simulations of radiatively driven convection: sensitivities to the representation of small scales. - J. Atmos. Sci. 56, 3963-3984.

STULL, R.B., 1988: An Introduction to Boundary Layer Meteorology. Kluwer Academic Publishers, Dordrecht,666 pp.

Willis, G.E., J.W. DEARDORFF, 1981: A laboratory study of dispersion from as source in the middle of the convectively mixed layer. - Atmos. Env. 15, 109-117.

WYNGAARD, J.C., R.A. BROST, 1984: Top-down and bottom-up diffusion of a scalar in the convective boundary layer - J. Atmos. Sci. 41, 102-112. 